\title{
EFECTO DE LA APLICACIÓN DE PRODUCTO COMERCIAL CON B+MO EN ÉPOCA TARDÍA DE FLORACIÓN DE Carica papaya EN CORRIENTES-ARGENTINA
}

\author{
The effect of applying a commercial product with B+Mo to Carica papaya \\ during late blooming in Corrientes, Argentina
}

\author{
Urán, Víctor J. ${ }^{1}$ Gaiad, José E. y ALAYÓN LUACES, Paula ${ }^{1}$ \\ ${ }^{1}$ Cátedra de Fruticultura, Facultad de Ciencias Agrarias Universidad Nacional del Nordeste. Sargento \\ Cabral 2131. Corrientes (3400). e-mail: palayonluaces@yahoo.com
}

\begin{abstract}
RESUMEN
La producción de mamón en zonas subtropicales tales como la región NEA, se ve limitada ya que el cultivo presenta anomalías ante la exposición a temperaturas menores a $16^{\circ} \mathrm{C}$. Las condiciones en la región incluyen estaciones de bajas temperaturas que coinciden con la floración y fructificación del mamón, lo cual hace que este cultivo sea destinado principalmente a la industria ya que no alcanza la maduración para consumo en fresco. El B es un micronutriente importante para la floración ya que participa en la germinación del polen y en el crecimiento del tubo polínico. El objetivo del presente trabajo fue evaluar el crecimiento y desarrollo vegetativo, fenológico y productivo de Carica papaya L. del cv Tainung 1 sometidos a un tratamiento con Sugar Mover ${ }^{\circledR}$ de Stoller (Boro 9\% + Molibdeno 0.008\%) en la floración de fines de verano. Se realizó la comparación entre dos tratamientos, un testigo sin aplicación y otro con una aplicación del producto comercial. Se evaluó el crecimiento vegetativo midiendo: altura de la planta, diámetro del tronco, número de hojas y se realizó el seguimiento fenológico, productividad y calidad de frutas. Se observó que las plantas testigo mostraron un retraso en la retención y crecimiento de frutos respecto a las tratadas con Sugar Mover ${ }^{\circledR}$. Si bien no se observaron diferencias en relación al crecimiento vegetativo entre tratamientos, si se observó que las plantas tratadas presentaron mayor cantidad de frutos por planta en relación a las plantas testigo. Se concluye que la utilización de Sugar Mover ${ }^{\circledR}$ en floración tardía presenta beneficios en cuanto al rendimiento y la posibilidad de adelantar la cosecha mercado, aunque no se observaron diferencias en la tolerancia a las bajas temperaturas.
\end{abstract}

Palabras claves: : azúcares, cultivo tropical, frío, microelementos, papaya, rendimiento.

\begin{abstract}
Papaya productivity in subtropical areas such as the Argentine Northeast (NEA) region is limited as it presents anomalies under exposure to temperatures below $16^{\circ} \mathrm{C}$. This region includes seasons with low temperatures that coincide with papaya flourishing and fruiting periods, consequently during cold seasons papaya stock is mainly reserved for the industry as it does not reach ripeness for fresh consumption. Boron is a micronutrient involved in flowering as it participates in pollen germination and pollen tube growth. The aim of this work was to assess the vegetative behavior phenology and production of Carica papaya L. cv Tainung 1, subjected to Sugar Mover ${ }^{\circledR}$ Stoller treatment (Boron 9\% + Molybdenum $0.008 \%$ ) during blossoming by the end of the summer. Two experimental plots were carried out, one with the commercial product and the other without it. Vegetative parameters (plant height, leaves number, stem diameter), fruit quality, yield and phenology were monitored and assessed. The control treatment showed retardancy in fruit retention and growth compared to those treated with Sugar Mov-er®. No differences were observed in relation to vegetative parameters between treatments. The treated plants presented a greater yield (fruits per plant) than control plants. It can be concluded that the use of Sugar Mover ${ }^{\circledR}$ presents benefits in terms of yield and the possibility to obtain the first fruits in the mar-ket although no differences in tolerance to low temperatures were observed.
\end{abstract}

Key words: chilly weather, microelements, papaya, sugars, tropical crop, yield.

Recibido: 5/Ago/2020. Aceptado: 9/Abr/2021 


\section{INTRODUCCIÓN}

Carica papaya L., comúnmente conocida como mamón, papaya, mamao, lechosa, pertenece al orden Parietales familia Caricácea, es una planta arborescente perennifolia, cuya copa es abierta y redondeada y las hojas son simples de pecíolo largo.

La producción de mamón en Argentina alcanza las 4100 Tn, provenientes de 369 ha totales, siendo Misiones la principal provincia productora (295 ha) (Sánchez, 2020). Sin embargo, las provincias de Corrientes, Misiones y Formosa se registran sectores productivos asociados a pequeñas empresas.

El fruto del mamón es valorado para su consumo en fresco por su sabor y por su alto valor nutritivo y aporte de vitaminas y minerales (Alonso et al., 2008), sin embargo también puede ser aprovechado con fines industriales y medicinales para extracción de papaína, una enzima proteolítica muy apreciada para ablandar carnes y para aclarar bebidas.

Por ser un cultivo de origen tropical, las condiciones agroecológicas más favorables para su desarrollo y crecimiento son aquellas en las que se registran altas insolaciones, ausencia de heladas y pluviometrías de alrededor de $1300 \mathrm{~mm}$ anuales. Según Knight (1980) el óptimo desarrollo y crecimiento del mamón se obtiene entre $21^{\circ} \mathrm{C}$ y $33^{\circ} \mathrm{C}$, clasificándose a este cultivo como una especie tropical sensible a las bajas temperaturas (Ogden et al., 1981). Dentro de la región del nordeste Argentino (NEA) contamos con zonas aptas para el cultivo de mamón aunque las condiciones durante el invierno, coincidentes con el desarrollo y maduración de los frutos, hacen que estos cultivos sean destinados principalmente a la industria debido a que no alcanzan madurez fisiológica en la planta al inicio de la temporada de bajas temperaturas. Cuando las temperaturas descienden por debajo del óptimo y según sea el momento del ciclo del cultivo se pueden presentar anomalías. Galán Saúco y Rodríguez Pastor (2007) señalan que temperaturas por debajo de los $18^{\circ} \mathrm{C}$ provocan carpeloidía de los frutos (conocidos como cara de gato sin valor comercial), cambios de género de la flor, reducción de la viabilidad del polen y disminución del contenido total de azúcares de los frutos.

En las plantas el elemento Boro (B) está involucrado en tres procesos principales que incluyen: preservación de la estructura de la pared celular, mantenimiento de las funciones de la membrana y cofactor de las actividades metabólicas (Malavé Acuña y Carrero Molina, 2007). Hay antecedentes del rol del B en relación al estrés por bajas temperaturas en especies de clima tropical (Huang et al, 2005). Dordas y Brown (2000), demostraron que las diferentes proporciones de esteroles y ácidos grasos de cadenas más largas en la membrana plasmática de células de raíz cambia significativamente el consumo de B en mutantes de Arabidopsis thaliana y relacionaron estos cambios a diferentes coeficientes de permeabilidad para el ácido bórico a través de membranas plasmáticas que contienen diferentes grupos de lípidos y ácidos grasos. El descenso del nivel de esteroles en la membrana plasmática puede incrementar su fluidez y permeabilidad al agua y a iones, lo cual está correlacionado con la tolerancia de la planta al frío (Hugly et al., 1990), siendo una respuesta común el incremento de la rigidez en la membrana en especies susceptibles a las bajas temperaturas (Queiroz et al., 1998). Sumado a ello, se reconoce la acción de este nutriente como elemento que dentro de la planta interviene en la translocación de los azúcares, el B es móvil en el floema de aquellas especies que utilizan polioles (azúcares simples: manitol, sorbitol) como un metabolito fotosintético primario con alta afinidad para enlazar al B para su posterior transporte en el floema hacia zonas de acumulación activa, como los meristemas vegetativos o reproductivos (Brown et al., 2008; Brown y Hu, 1996; 1998; Hu y Brown, 1997). En frutales, tales como palta, manzanas, almendros y citrus (Robbertse et al., 1992; Fang et al., 2019; Nyomora et al., 2000; Ullah et al., 2012) es ampliamente difundida la importancia del B en la floración ya que este elemento es clave en la germinación del polen y el crecimiento del tubo polínico, lo que se puede traducir en un aumento de la producción de frutos (Lovatt y Dugger, 1994; Wojcik y Wojcik, 2006; Gowhar, 2017). Sumado a ello existen evidencias de que la aplicación de B en primavera en plantas no deficientes de este elemento (basado en análisis foliares) ha sido eficiente para aumentar el cuajado de frutos de palta (Robbertse et al., 1992).

El formulado comercial, Sugar Mover ${ }^{\circledR}$ de Stoller, contiene Boro 9\% + Molibdeno 0.008\% y se indica como fertilizante inorgánico para revertir el movimiento de los azúcares, favoreciendo su transporte desde el follaje hacia los frutos, tallos y demás órganos a cosechar, incrementando las características de calidad, tales como calibre, uniformidad del llenado, grados Brix, contenido de almidones y sólidos totales, concentración de fenoles y taninos entre otros, así como reduce los desórdenes fisiológicos y malformaciones de los frutos. 
También se menciona que una de las ventajas del uso del mismo seria prevenir el aborto y la reabsorción de frutos, además de uniformizar el llenado de los frutos (Stoller, 2021). En el caso en particular del mamón, está indicada su aplicación luego del inicio de floración, sin embargo esta información es originaria de zonas tropicales sin antecedentes de su comportamiento en regiones subtropicales.

Según Campostrini y Glenn (2007) un desafío para la producción del cultivo de mamón es incrementar la calidad de la fruta cuando se cultivan fuera de los sitios tradicionales de producción, donde los factores abióticos son limitantes, ese es el caso del NEA donde las bajas temperaturas de otoño e invierno inciden directamente en la calidad de fruta para su aprovechamiento en fresco.

Conocer el comportamiento de esta especie en las condiciones agroecológicas de una determinada región provee de bases científicas para desarrollar estrategias de manejo adecuadas para obtener una buena productividad y calidad de fruta. Las condiciones ambientales inciden en el crecimiento, desarrollo, productividad y calidad del mamón por lo que ajustar practicas agronómicas en cada región es un aporte para el conocimiento e implantación del cultivo en cada zona, en búsqueda de mejorar la producción.

El objetivo del presente trabajo fue evaluar el desarrollo vegetativo, fenológico y productivo de Carica papaya L. del cv Tainung 1 sometido a un tratamiento con Sugar Mover ${ }^{\circledR}$ de Stoller (Boro 9\% + Molibdeno $0.008 \%$ ) en la floración de fines de verano en las condiciones agroecológicas de Corrientes Argentina.

\section{MATERIALES Y MÉTODOS}

\section{Características del Predio}

El ensayo se llevó a cabo en un lote experimental de $32 \mathrm{~m}^{2}$ ubicado en el Campo Didáctico Experimental de la Facultad de Ciencias Agrarias de la Universidad Nacional del Nordeste (CDEA), ubicado sobre la ruta Nacional 12 km 1031 (Latitud Sur: 27o 28' 27", Longitud Oeste: 58o 47' 00"); altura sobre el nivel del mar 70 msnm Provincia de Corrientes, Argentina.

El suelo del sitio de experimentación ha sido clasificado como Udipsament acuico hipertérmico de la serie Ensenada Grande. Presenta un pH de 5,28 en los primeros $20 \mathrm{~cm}$ y de 4,76 entre los $20 \mathrm{~cm}$ y $40 \mathrm{~cm}$. Su baja fertilidad natural y susceptibilidad a la erosión, ubica a éstos suelos en Subclase II y III (Escobar et al., 1994). El relieve es suavemente ondulado, con pendientes de 1 a 1,5\%. Las características de estos suelos son de baja fertilidad natural, con muy poco contenido de materia orgánica los cuales se asocian a deficiencias de nutrientes (Tabla 1). El clima se caracteriza por presentar precipitaciones promedio de $1300 \mathrm{~mm}$ anuales, evapotranspiración media anual según Thornthwaite de $1100 \mathrm{~mm}$ y una temperatura media anual de $21,6^{\circ} \mathrm{C}$, con un período libre de heladas de 340 a 360 días. La región se clasifica como un clima mesotermal, cálido templado, sin estación seca, con precipitaciones máximas en otoño y veranos muy cálidos (media superior a los $18^{\circ} \mathrm{C}$ ) clasificado, según Copen como Climas Templados Húmedos (De Fina y Ravelo, 1985).

En el lote en estudio se realizaron aplicaciones de fertilización y productos agroquímicos para el manejo sanitario de manera que las plantas en estudio no tengan limitantes. El programa de fertilización fue de $120 \mathrm{~kg}$ de $\mathrm{N} ; 30 \mathrm{~kg}$ de fósforo $\left(\mathrm{P}_{2} \mathrm{O}_{5}\right)$ y $100 \mathrm{~kg}$ de potasio $\left(\mathrm{K}_{2} \mathrm{O}\right)$ por ha distribuidos desde plantación en septiembre hasta finales del ensayo en agosto. La distribución acompañó el desarrollo de las plantas, hasta floración se agregó el $30 \%$ del N y del P y el $20 \%$ del K, y luego se distribuyó el resto cada 45 días en cantidades iguales.

Tabla 1: Análisis de suelo del sitio de experimentación

\begin{tabular}{cccccccc}
\hline & $\mathbf{p H}$ & $\mathbf{M O}$ & $\mathbf{N}$ & $\mathbf{P}$ & $\mathbf{K}$ & $\mathbf{C a}$ & $\begin{array}{c}\text { Mg } \\
\mathrm{meq} / 100 \mathrm{~g}\end{array}$ \\
\hline $\begin{array}{c}\text { Serie Ensenada } \\
\text { Grande }\end{array}$ & 7,3 & 0,24 & 0,023 & 13 & 0,14 & $<1$ & $<1$ \\
\hline
\end{tabular}

\section{Material vegetal y tratamientos}

El ensayo se realizó en plantas de mamón Carica papaya L. del cv Tainung 1. El 26 de septiembre de 2016 se implantaron plantines de 2 meses de edad en condiciones de campo natural a un distanciamiento de $2 \mathrm{~m}$ por $2 \mathrm{~m}$ en una distribución de tres bolillos (2906 plantas ha $\left.{ }^{-1}\right)$ con riego por goteo. 
El cultivar Tainung perteneciente al grupo Formosa, se caracteriza por alcanzar los $2 \mathrm{~m}$ de altura aproximadamente y una altura de cargue de 55 a $60 \mathrm{~cm}$ (altura de inserción del primer fruto). Presenta un tronco recto y cilíndrico con un diámetro entre 20 y $30 \mathrm{~cm}$. Sus frutas hermafroditas (alargados) presentan pulpa de color naranja al madurar con un peso promedio de $1,1 \mathrm{~kg}$ y una dulzura de $12,8^{\circ}$ Brix (Morais de Lima et al., 2009). El tiempo a cosecha en zonas tropicales es de 6 a 7 meses después de trasplante, pudiéndose realizar durante 7 meses. Bajo condiciones estándar de producción puede alcanzar 120 toneladas aproximadamente por hectárea.

El diseño experimental fue completamente al azar, la unidad experimental consistió en una planta con 7 plantas por tratamiento.

Durante la etapa de la floración ya avanzada (10 de marzo) se aplicaron los siguientes tratamientos del formulado comercial a base de $9 \%$ de B $+0,008 \%$ de Mo (Sugar Mover ${ }^{\circledR}$ Stoller) y testigo sin aplicación de producto comercial:

1) Sin aplicación Sugar Mover ${ }^{\circledR}$ Stoller (S)

2) Con aplicación Sugar Mover ${ }^{\circledR}$ Stoller (C)

La aplicación del producto se realizó con mochila manual a partir del 6 de marzo (60 días después del inicio la floración) cada 15 días y hasta el 30 de mayo, pulverizando toda la planta con la solución preparada al $2 \%$ según marbete.

\section{Variables evaluadas}

Para analizar la evolución de las plantas se realizaron mediciones de crecimiento y desarrollo, semanalmente se examinaron el total de plantas por tratamiento y en las mismas se determinó:

a. Altura de planta $(\mathrm{cm})$ : esta medida se tomó desde la base del tallo hasta el ápice.

b. Número de hojas: se contaron todas las hojas de cada planta.

c. Diámetro de tronco $(\mathrm{cm})$ : con calibre se midió el diámetro de tallo a $10 \mathrm{~cm}$ del suelo.

e. Números de frutos: se contabilizó la totalidad de frutos por planta.

g. Biometría de frutos al momento de cosecha en tres frutos marcados por planta provenientes de la floración de marzo se midió:

g1. Peso (g): peso individual de los frutos.

g2. Longitud y diámetro de cada fruto y cavidad ovárica en cm utilizando calibre digital.

g3. Volumen de los frutos $\left(\mathrm{cm}^{3}\right)$ : volumen de agua desplazada por el fruto en un recipiente graduado.

Las variables de crecimiento y desarrollo de los frutos se comenzaron a medir cuando se visualizaron los primeros frutos fijados (al detectarse crecimiento de estos) provenientes de las flores abiertas desde los primeros días de marzo.

i: Registro fenológico: se observó el desarrollo de la planta tomando registro de los estadios en los cuales se encontraba cada planta. Los registros fenológicos comenzaron a tomarse en el momento que se visualizaron los primeros pimpollos (inicio de floración). Se consideró inicio de fase cuando el $50 \%$ de los individuos se encontraban en dicho momento.

j. Registros meteorológicos: Temperaturas diarias cada 10 minutos, precipitación y humedad relativa ambiente con estación meteorológica.

Los resultados fueron analizados estadísticamente utilizando test de T Student para muestras independientes como prueba univariada de cada una de las variables. Además se realizó un análisis exploratorio multivariado de todos las variables utilizando el programa Infostat 2018 (Di Rienzo et al., 2018)

\section{RESULTADOS Y DISCUSIÓN}

Las variables vegetativas evaluadas en este ensayo para ambos tratamientos durante todo el ciclo productivo indicaron que la aplicación de Sugar Mover ${ }^{\circledR}$ no presentó diferencias significativas ( $\mathrm{p}$ valor $\leq 0,05$ ) en ninguna de estas variables como ser altura de planta, diámetro de tallo y número de hojas. La altura de plantas (Fig. 1) alcanzó a un valor máximo promedio de plantas del lote de $1,38 \mathrm{~m}$ y en cuanto al diámetro de tallo el valor máximo promedio medido fue de $8,61 \mathrm{~cm}$ (Fig. 2). 


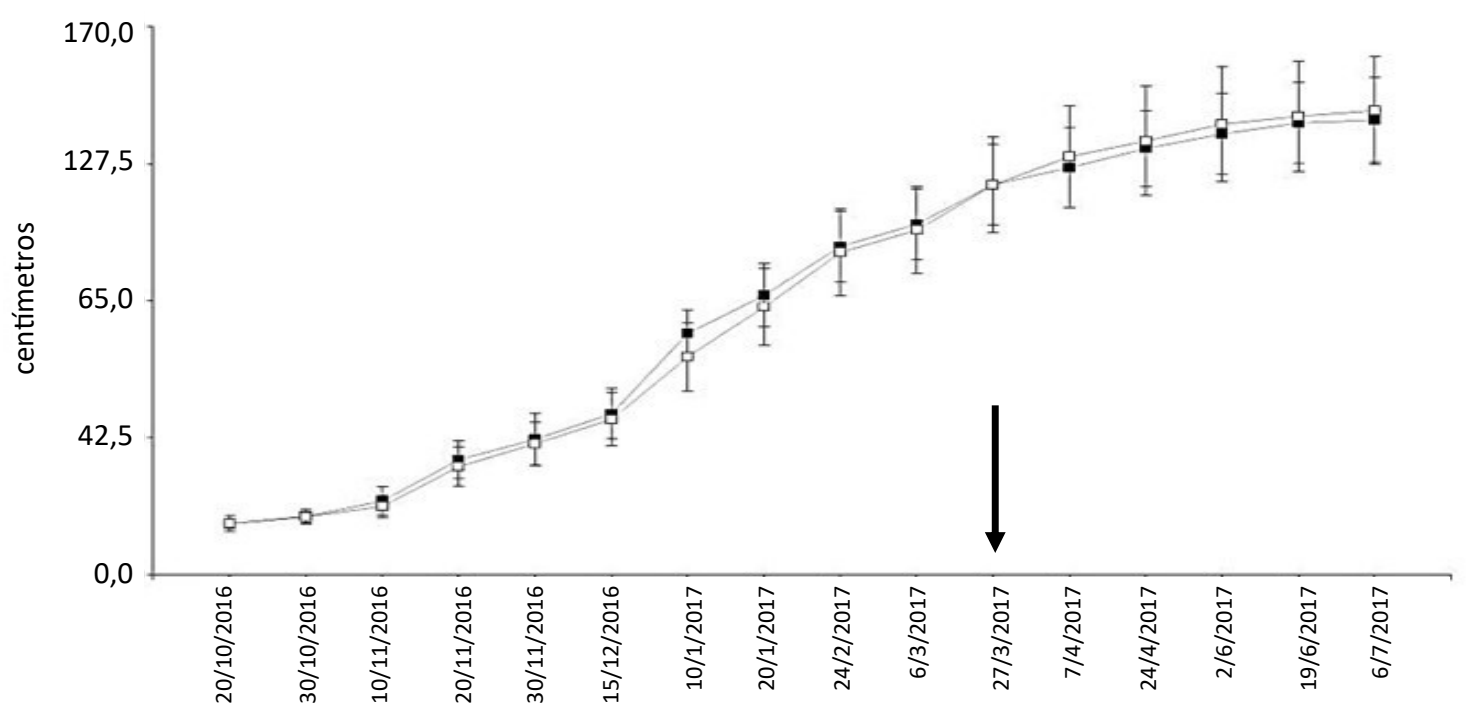

Figura 1. Altura de plantas de mamón con (ロ) y sin ( $\square$ ) aplicación de B+Mo de Sugar Mover $®$ (flecha indica inicio de aplicaciones).

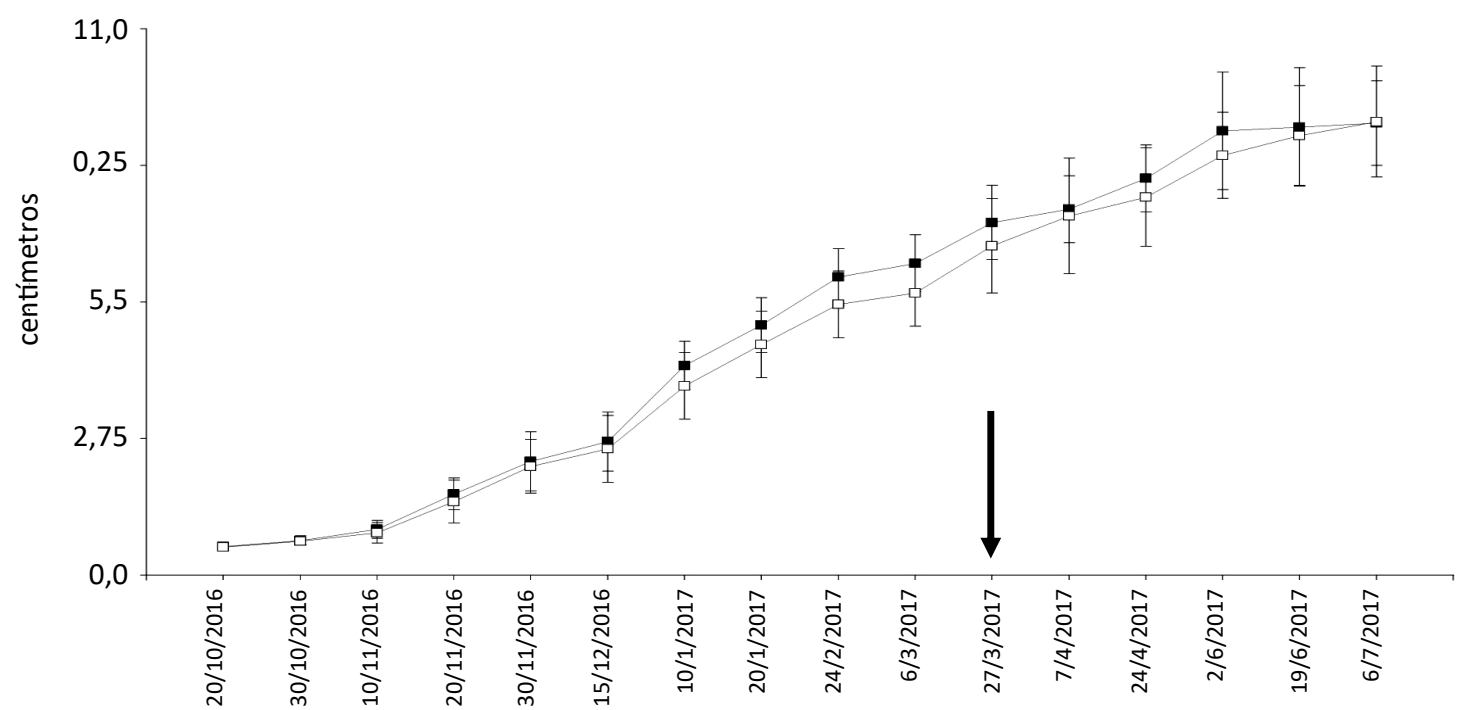

Figura 2. Diámetro de tallo de plantas de mamón con (a) y sin ( $\square$ ) aplicación de B+Mo de Sugar Mover $\mathbb{R}$ (flecha indica inicio de aplicaciones).

El recuento de las hojas no presentó diferencias significativas entre ambos tratamientos (Fig. 3), el aumento de las mismas se mantuvo constantes a partir de abril, coincidente con la época del año en la cual se empezaron a registrar temperaturas medias mensuales promedio de $21,09^{\circ} \mathrm{C}$ (Fig. 4), las cuales ya incidieron en claramente en este parámetro.

Las variables evaluadas de desarrollo vegetativo para este cultivar en las condiciones subtropicales del ensayo difirieron de lo citado por Alonso et al. (2009) para el cultivar Tainung 1 quien establece que el mismo puede llegar a una altura aproximada entre 2,81 - 2,84 m, presentar un diámetro promedio de $13-15 \mathrm{~cm}$ y entre 66 - 81 hojas en todo el ciclo, sin embargo, esta información es proveniente de zonas tropicales con ambientes más propicios para el desarrollo de esta especie. Los valores máximos alcanzados en nuestra región antes del descenso de temperatura y en relación a las variables vegetativas representan aproximadamente un $60 \%$ menos de altura, un $40 \%$ menos en el diámetro de tallo y $54 \%$ menos de hojas que el potencial esperado para la variedad. Esta disminución está directamente asociada a las temperaturas requeridas por el cultivo. El rango óptimo de temperatura para que el mamón tenga un buen desarrollo es de $24-26^{\circ} \mathrm{C}$ con mínimas medias anuales superiores a $18^{\circ} \mathrm{C}$, por debajo de esta temperatura, las plantas retardan su crecimiento (Bhattarai et al., 2004). 


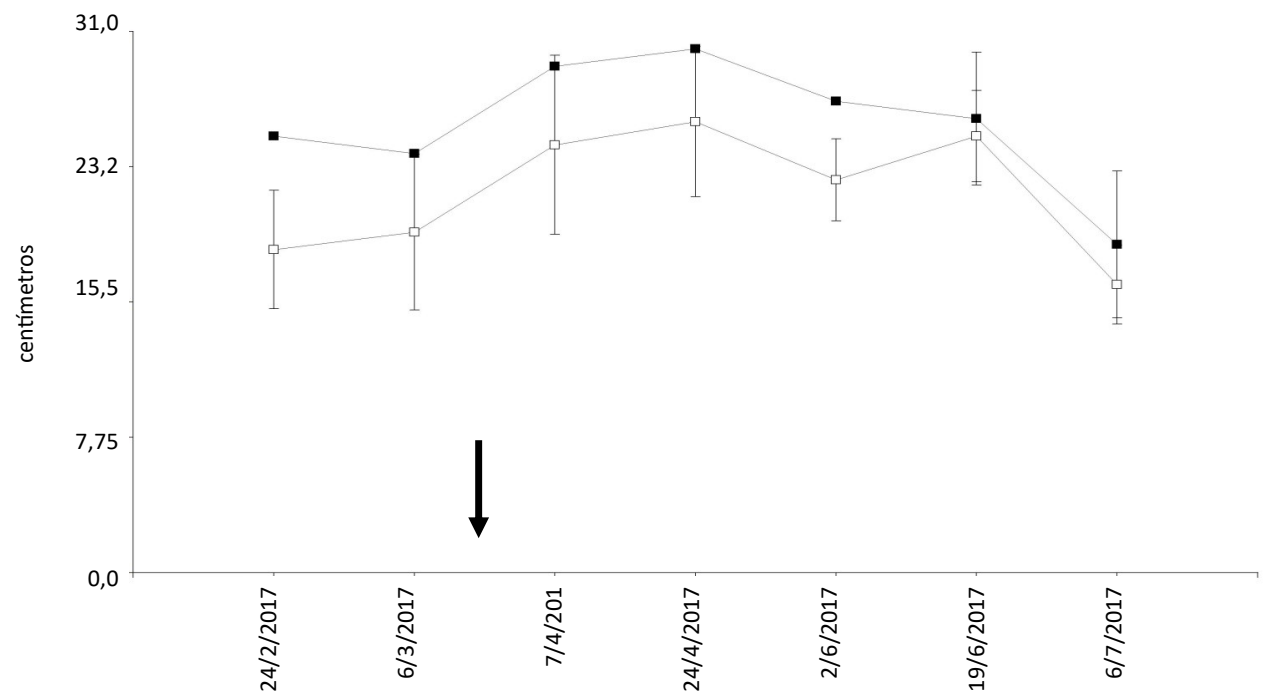

Figura 3. Número de hojas de plantas de mamón con (ロ) y sin ( $\square$ ) aplicación de B+Mo de Sugar Mover ${ }^{\circledR}$ (flecha indica inicio de aplicaciones).

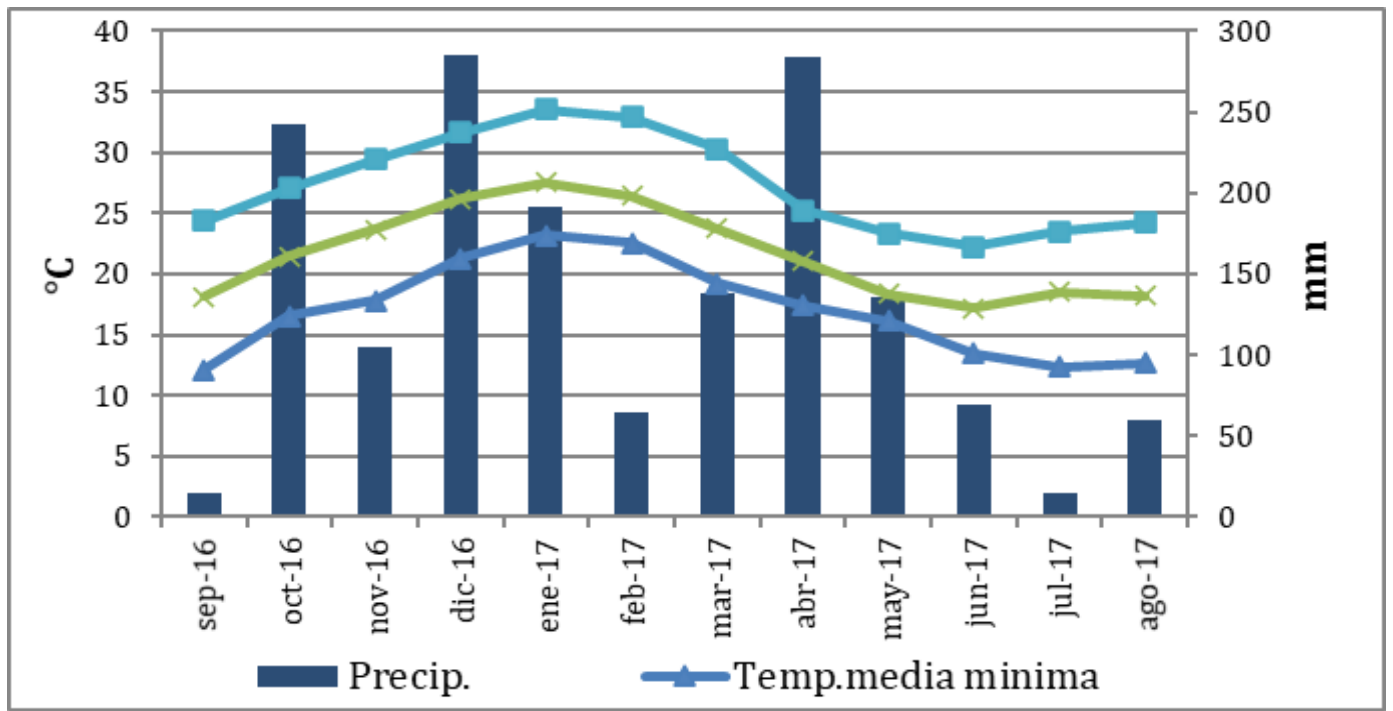

Figura 4. Climograma durante meses de crecimiento y desarrollo de plantas de mamón en Corrientes

Argentina (2016-2017)

De allí y en relación al desarrollo vegetativo alcanzado por las plantas de Tainung en esta región, las temperaturas medias optimas recién se alcanzan en forma constante a partir de diciembre y hasta marzo que vuelven a descender por debajo del óptimo. Estos resultados coinciden con los citados por Allan (2002) quien indica que en condiciones subtropicales el cultivo de papaya (mamón) se desarrolla bien sólo durante cuatro meses en el año.

La etapa vegetativa del mamón en las condiciones de este ensayo fue de 116 días, los registros fenológicos se presentan en la Tabla 1, donde se observa que el inicio de floración en todas las plantas se registró desde el 10 de enero. La primera aplicación del producto Sugar Mover ${ }^{\circledR}$, se realizó el día 7 de marzo del 2017 aproximadamente a los 60 días después del inicio de floración, a fines de evaluar su efecto en la floración de fines de verano y otoño. En frutales donde el B es inmóvil, pero esencial para el proceso de floración, las aplicaciones son efectivas directamente en los botones o en las flores (Brown y Hu, 1998). Estudios previos no son concluyentes respecto a la movilidad del B en plantas de mamón (Malavé Acuña y Carrero Molina, 2007).

Hasta la fecha de aplicación del producto el lote se encontraba con fenología homogénea. Las plantas tratadas respecto a las no tratadas se adelantaron a partir de los estadíos iniciales de desarrollo de los frutos, 24 y 28 días en cuajado y fructificación respectivamente (Tabla 1). 
Los estadios de desarrollo de frutos provenientes de estas floraciones se dieron en los meses de marzo y abril, en donde las temperaturas mínimas medias mensuales registradas fueron de $18,45^{\circ} \mathrm{C}$ y $17,42^{\circ} \mathrm{C}$ respectivamente, las cuales se encontraban en el límite inferior de tolerancia del cultivo. De allí a que la aplicación del tratamiento con $\mathrm{B}+$ Mo pudo haber incidido en el adelanto de estos estadios por su acción beneficiosa para la floración y también por su efecto protector ante condiciones de bajas temperaturas. Si bien no se puede descartar que el producto comercial haya favorecido a la planta desde el punto de vista nutricional hay antecedentes que citan que la aplicación de boro durante la floración en papaya aumenta el crecimiento del tubo de polen e incide positivamente en el desarrollo de las etapas de floración y fructificación (Janaki et al., 2020).

En la figura 5, se presenta la variación de la cantidad de frutos totales promedios para ambos tratamientos. Las plantas sin tratamiento presentaron menor cantidad de frutos con diferencia significativa respecto de aquellas que fueron pulverizadas con Sugar Mover ${ }^{\circledR}$. El máximo recuento de frutos provenientes de la floración desde que se iniciaron los tratamientos fue en el mes de junio con 18,5 frutos promedio en plantas tratadas con $\mathrm{B}+\mathrm{Mo}$, mientras que aquellas no tratados solo alcanzaron a 7,90 frutos promedio por planta. Luego de esta fecha la cantidad de frutos se vio comprometida ya que en la segunda semana del mes de julio se registraron heladas agrometeorológicas y meteorológicas.

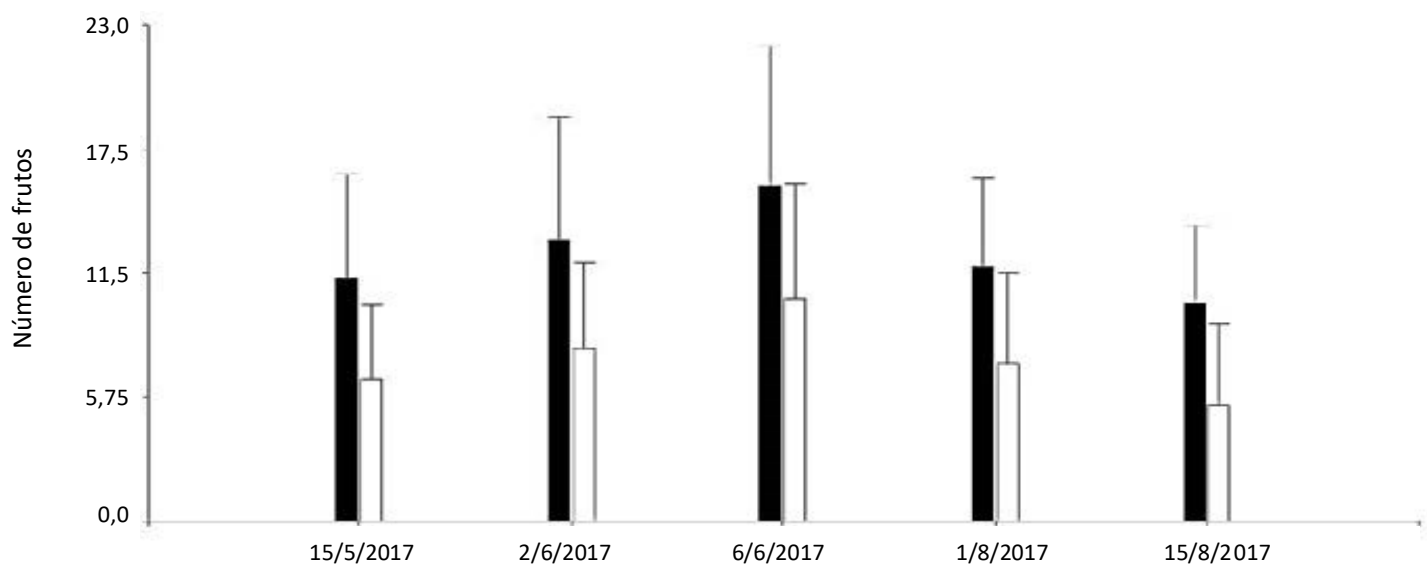

Figura 5. Número de frutos por planta de mamón con (•) y sin (口) aplicación de B+Mo de Sugar Mover ${ }^{\circledR}$.

La mayor retención de frutos en plantas tratadas puede atribuirse a que éstas tuvieron mayores posibilidades de fecundación y posterior cuaje, ya que según Lewis (1980), Majewska-Sawka y Nothnagel (2000), una adecuada disponibilidad de Boro asegura una buena germinación del polen y ayuda a la formación del tubo polínico tanto en el estilo como el estigma. Esta fecundación favoreció la retención anticipada de los frutos en comparación con los no tratados, los cual se reflejó en un adelanto de 24 y 28 días en cuajado y fructificación respectivamente (Tabla 2).

Tabla 2: Seguimiento fenológico de plantas de mamón con y sin aplicación de B+Mo de Sugar Mover ${ }^{\circledR}$ (flecha indica inicio de aplicaciones).

\begin{tabular}{|c|c|c|c|c|c|c|c|c|c|c|c|c|}
\hline $\begin{array}{l}\text { Estadio } \\
\text { fenológico }\end{array}$ & $10 / 01$ & $25 / 02$ & $06 / 03$ & $20 / 03$ & $30 / 03$ & $17 / 04$ & $24 / 04$ & $15 / 05$ & $02 / 06$ & $19 / 06$ & $06 / 07$ & $26 / 07$ \\
\hline Inicio Floración & - & -- & & & & & & & & & & \\
\hline Plena Floración & & & - & & & & & & & & & \\
\hline Cuajado & & & & & - & - & -- & & & & & \\
\hline $\begin{array}{c}\text { Crecimiento } \\
\text { frutos }\end{array}$ & & & & & & & & - & -- & - & -- & -- \\
\hline & Con ar & licación & - & - & & & Sin ap & icación & -- & - & & \\
\hline
\end{tabular}


Las variables físicas de calidad de frutos cuantificadas al momento de la cosecha se presentan en la Tabla 3. El peso medio máximo obtenido fue de $858,1 \mathrm{~g}$, la mayor longitud fue de $18,73 \mathrm{~cm}$ y un diámetro de $10,23 \mathrm{~cm}$, todos ellos en frutas provenientes de plantas tratadas. Las determinaciones de las variables físicas no presentaron diferencias significativas entre tratamientos. Los valores de biometría de los frutos de esta variedad fueron diferentes a lo que menciona Alonso et al. (2009), quienes dicen que en condiciones óptimas los frutos de Tainung 1 pueden alcanzar un peso de 1,4-1,6 kg, llegar a un largo entre $37-39 \mathrm{~cm}$ y hasta un diámetro mayor de $32 \mathrm{~cm}$. El desarrollo de frutos se vio afectado por las bajas temperaturas, principalmente en los meses de mayo y junio, lo cual incidió muy fuertemente en esta variable independientemente de los tratamientos. Las frutas de papaya presentan el patrón general de crecimiento sigmoide para aumentar el volumen, pero la forma de la curva varía considerablemente según las condiciones ambientales en el mes de cuajado y el clon. Las temperaturas medias semanales, por debajo de aproximadamente $19{ }^{\circ} \mathrm{C}$, hacen que las etapas iniciales y finales de crecimiento relativo de la fruta sean más prolongadas (Allan, 2002).

Tabla 3: Biometría de frutos de mamón con y sin aplicación de B+Mo de Sugar Mover ${ }^{\circledR}$ (promedio \pm desvío estándar).

\begin{tabular}{cccccc}
\hline Tratamiento & $\begin{array}{c}\text { Largo } \\
(\mathbf{c m})\end{array}$ & $\begin{array}{c}\text { Diámetro } \\
\mathbf{( c m})\end{array}$ & Peso $(\mathbf{g})$ & $\begin{array}{c}\text { Volumen } \\
(\mathbf{m L} \mathbf{)}\end{array}$ & $\begin{array}{c}\text { Cavidad Ovárica } \\
(\mathbf{c m})\end{array}$ \\
\hline Con B+Mo & $18,73 \pm 4,1$ & $10,23 \pm 2,7$ & $858,1 \pm 224$ & $1237,5 \pm 331,5$ & $0,61 \pm 0,11$ \\
Sin B+Mo & $17,3 \pm 4,4$ & $9,83 \pm 1,9$ & $761,17 \pm 254$ & $1154,17 \pm 253$ & $0,45 \pm 0,17$ \\
\hline
\end{tabular}

Los rendimientos en toneladas de fruta por hectárea se estimaron a partir de la máxima cantidad promedio de frutos por planta y el peso promedio para ambos tratamientos. Teniendo en cuenta el marco de plantación utilizado, las plantas con Sugar Mover ${ }^{\circledR}$ obtuvieron 46,12 $\mathrm{Tn} \mathrm{ha}^{-1}$, en cuanto a las plantas testigo su rinde alcanzó las 17,5 $\mathrm{Tn} \mathrm{ha}^{-1}$, en ambos casos valores por debajo de lo esperado para la variedad en zonas tropicales.

\section{CONCLUSIONES}

La utilización de producto comercial con B+Mo (Sugar Mover ${ }^{\circledR}$ ) en el cultivo de mamón (Carica papaya L.) en floración de fines de verano y en las condiciones subtropicales de este ensayo, presentó beneficios aumentando el rendimiento y por la posibilidad de adelantar la cosecha de frutas.

El aumento de rendimiento en plantas tratadas estuvo relacionado a una mayor cantidad de frutos por plantas más que a características biométricas de los frutos.

\section{REFERENCIAS}

Allan, P. (2002). Carica papaya responses under cool subtropical growth conditions. Acta Horticulturae. 575: 757-763. https://doi.org/10.17660/ActaHortic.2002.575.89

Alonso, M.; Tornet, Y.; Ramos, R.; Farrés, E.; Castro, J. y Rodríguez, M.C. (2008). Evaluación de tres cultivares de papaya del grupo Solo basada en caracteres de crecimiento y productividad. Cultivos Tropicales 29 (2): 59-64.

Alonso, M.; Tornet, Y.; Ramos, R.; Farrés, E.; Castro, J. y Rodríguez, M.C. (2009). Evaluación de dos híbridos de papaya introducidos en Cuba. Agronomía Costarricense 33(2): 267-274.

Bhattarai, K.R.; Vetaas, O.R. y Grytnes, J.A. (2004). Fern species richness along a central Himalayan elevational gradient, Nepal. J. Biogeogr 31: 389-400.

Brown, P.H. y Hu, H. (1996). Phloem mobility of boron in species dependent: evidence for phloem mobility in sorbitol-rich species. Ann. Bot., 77(5): 497-505. https://doi.org/10.1006/anbo.1996.0060

Brown, P.H. y Hu, H. (1998). Boron mobility and consequent management in different crops. Better Crops 82 (2): 28-31.

Brown, P. H.; Bellaloui, N.; Wimmer, M. A.; Bassil, E. S.; Ruiz, J.; Hu, H.; Pfeffer, H.; Dannel, F. y Römheld, V. (2008). Boron in plant biology. Plant Biol. 4 (2): 205-223. https://doi.org/10.1055/s-2002-25740

Campostrini, E. y Glenn; D.M. (2007) Ecophysiology of papaya: a review. Braz. J. Plant Physiol., 19(4):413424. https://doi.org/10.1590/S1677-04202007000400010

De Fina, A.L. y Ravelo, A.C. (1985). Climatología y Fenología Agrícolas. 4ta Ed. EUDEBA. Buenos Aires, Argentina. 354 pp. 
Di Rienzo, J.A.; Casanoves, F.; Balzarini, M.G.; González, L.; Tablada, M. y Robledo, C.W. (2018). InfoStat versión 2012. Grupo InfoStat, FCA, Universidad Nacional de Córdoba, Argentina. Disponible en: http:// www.infostat.com.ar

Dordas, C. y Brown, P.H. (2000). Permeability of boric acid across lipid bilayers and factors affecting it. J. Membr. Biol. 175 (2): 95-105. https://doi.org/10.1007/s002320001058

Escobar, E.H; Ligier, D.; Melgar, R.; Matteio, H. y Vallejos, O. (1994). Mapa de Suelo de los Departamentos Capital, San Cosme e Itatí, de la Provincia de Corrientes. INTA - CFI - ICA 125 pp.

Fang, F.; Du, B.S.; Zhang, Q.; Xing, Y.; Cao, Q.Q. y Qin, L.K. (2019). Boron deficiency alters cytosolic $\mathrm{Ca} 2+$ concentration and affects the cell wall components of pollen tubes in Malus domestica. Plant Biology 21 (2): 343-351. https://doi.org/10.1111/plb.12941

Galán Saúco, V.G. y Rodríguez Pastor, M.C.R. (2007). Greenhouse cultivation of papaya. Acta Hort. 740:191195. https://doi.org/10.17660/ActaHortic.2007.740.22

Gowhar A.D. (2017). Impact of Boron Nutrition in Fruit Crops. Int. J. Curr. Microbiol. App. Sci. 6(12): 4145 4155. https://doi.org/10.20546/ijcmas.2017.612.476

Hu, H. y Brown, P. (1997). Absorption of boron by plant roots. Plant Soil 193 (1- 2): 49-58. https:// doi.org/10.1023/A:1004255707413

Huang, L.B.; Ye, Z.Q.; Bell, R.W. y Dell, B. (2005). Boron nutrition and chilling tolerance of warm climate crop species. Ann. Bot. 96 (5) 755-767. https://doi.org/10.1093/aob/mci228

Hugly, S.; McCourt, P.; Browse, J.; Patterson, G. W.; Somerville, C. (1990). A chilling sensitive mutant of Arabidopsis with altered steryl-ester metabolism. Plant Physiol. 93 (3): 1053-1062. https:// doi.org/10.1104/pp.93.3.1053

Janaki, D.; Chitra, R.; Prabhu, S.y Poorniammal, R. (2020). Boron Nutrition in Fruit Crops. Biotica Research Today 2(5): 241-244. https://bioticainternational.com/ojs/index.php/biorestoday/article/view/143

Knight, R.J. (1980). Origin and world importance of tropical and subtropical fruit crops. En: S. Nagy y P.E. Shaw (Eds.), Tropical and Subtropical Fruits: Composition, Properties and Uses. Pp.1-120. AVI Publishing, Westport.

Konsaeng, S.; Dell, B. y Rerkasem B. (2005). A Survey of Woody Tropical Species for Boron Retranslocation, Plant Production Science. 8 (3): 338-341. https://doi.org/10.1626/ps.8.338

Lewis, D.H. (1980). Are there any inter-relations between the metabolic role of boron, synthesis of phenolic phytoalexins and the germination of pollen?. New Phytol. 84 (2): 261-270. https://doi.org/10.1111/ j.1469-8137.1980.tb04426.x

Lovatt, C.J. y Dugger, W.M. (1984). Boron. En: E, Frieden (Ed.). Biochemistry of the Essential Ultratrace Elements. Biochemistry of the Elements, vol 3. Springer, Boston, MA. https://doi.org/10.1007/978-1-46844775-0 17

Malavé Acuñā, A. C.y Carrero Molina, P.E. (2007). Desempeño funcional del boro en las plantas. Revista UDO Agrícola 7 (1): 1-14.

Majewska Sawka, A. y Nothnagel, E.A. (2000). The multiple roles of arabinogalactan proteins in plant development. Plant Physiol. 122 (1): 3-9. https://doi.org/10.1104/pp.122.1.3

Morais de Lima, L.; Dantas de Morais, P.L.; Valente de Medeiros, É.; Mendonça, V.; Ferreira Xavier, I.; Andrade Leite, G. (2009) Qualidade pós-colheita do mamão formosa 'Tainung 01' comercializados em diferentes estabelecimentos no Município de Mossoró-RNRev. Bras. Frutic. 31 (3): 902-906. Jaboticabal. https://doi.org/10.1590/S0100-29452009000300038

Nyomora, A.S.; Brown, P.H.; Pinney, K. y Polito, V.S. (2000). Foliar Application of Boron to Almond Trees Affects Pollen Quality, Journal of the American Society for Horticultural Science 125 (2): 265-270. https://doi.org/10.21273/JASHS.125.2.265

Ogden, M.A.H.; Jackson, L.K. y Campbell, C.W. (1981) Florida tropical fruit culture via master gardener. Proceedings of the Florida State Horticultural Society: 94: 222-225.

Queiroz, C.G.S.; Alonso, A.; Mares Guia, M.; Magalhaes, A.C. (1998). Chilling-induced changes in membrane fluidity and antioxidant enzyme activities in Coffea arabica L. roots. Biol. Plant. 41 (3): 403-413. https://doi.org/10.1023/A:1001802528068

Robbertse, P.J.; Coetzer, L.A. y Bessinger, F. (1992). Boron: uptake by avocado leaves and influence on fruit production. En: C.J. Lovatt,(Ed.). Proc II World Avocado Congress. orange, CA. pp. 173-178

Sánchez, E. (2020). Programa Nacional Frutales Superficie ocupada por plantaciones frutales en el país y cambios en su estructura productiva. INTA Ediciones EEA Balcarce. 25pp.

Stoller (2021) Boletín técnico Sugar Mover ${ }^{\circledR}$. Disponible en: https://stoller.com.ar/productos/sugar-mover/

Ullah, S.; Khan, A.S.; Malik, A.U.; Afzal, I.; Shahid M. y Razzaq, K. (2012). Foliar application of boron influences the leaf mineral status, vegetative and reproductive growth, yield and fruit quality of 'Kinnow' mandarin (Citrus reticulata Blanco). Journal of Plant Nutrition 35 (13): 2067-2079. https:// doi.org/10.1080/01904167.2012.717661

Wojcik, P. y Wojcik, M. (2006). Effect of boron fertilization on sweet cherry tree yield and fruit quality. Journal of plant Nutrition, 29: 1755-1766. https://doi.org/10.1080/01904160600897471 